\title{
Desarrollo de Competencias Docentes a partir de Metodologías Participativas Aplicadas a la Educación Ambiental
}

\author{
Mercedes Varela-Losada*, Uxío Pérez-Rodríguez, Francisco J. Álvarez-Lires, María M. Álvarez-Lires \\ Universidade de Vigo, Facultade de Ciencias da Educación e do Deporte, Campus da Xunqueira s/n, \\ Pontevedra-España (e-mail: mercedesvarela@uvigo.es, uxio.perez@uvigo.es, xabieral@uvigo.es, \\ lires@uvigo.es)
}

${ }^{*}$ Autor a quien debe ir dirigida la correspondencia

Recibido Jun. 30, 2014; Aceptado Ago. 04, 2014; Versión final recibida Oct. 16, 2014

\begin{abstract}
Resumen
Se analiza una propuesta de aprendizaje basada en metodologías participativas, cuyo fin es proporcionar al profesorado en formación inicial la oportunidad de trabajar en equipo y experimentar formas de enseñanza diferentes del modelo tradicional. Esto les permite reflexionar sobre su adecuación al proceso de aprendizaje y favorece el desarrollo de competencias docentes en el área de las ciencias experimentales y medioambientales. La integración de la educación ambiental en la escuela requiere enfoques metodológicos que impliquen una visión globalizada de la problemática ambiental, que fomenten la participación de los alumnos y la movilización de competencias transversales. Los resultados muestran que el alumnado valora positivamente metodologías como el aprendizaje basado en problemas o el trabajo cooperativo. Sin embargo, los futuros docentes deben mejorar sus conocimientos ambientales y su capacidad de analizar y reflexionar sobre la práctica docente, a fin de aplicar metodologías innovadoras en su futuro ejercicio profesional.
\end{abstract}

Palabras clave: educación ambiental, aprendizaje basado en problemas, aprendizaje cooperativo, formación del profesorado, competencias docentes

\section{Developing Teaching Competences through Participative Methodologies on Environmental Education}

\begin{abstract}
A proposal based on participatory methodologies, which aims to provide teachers in initial training the opportunity to work in a team, to experiment new methodologies and considering its use in the teaching and learning process. This contributes to promote the development of specific teaching skills in the area of experimental and environmental sciences. The integration of environmental education in schools requires methodological approaches that involve global vision of environmental problems, that promote the participation of students and that mobilize transversal skills. The results show that the students appreciate the innovative methodologies such as problem-based learning or cooperative work. However, future teachers should improve their knowledge in environmental topics and their ability to analyze and meditate on the teaching practice, to apply innovative methodologies in their future teaching career.
\end{abstract}

Keywords: environmental education, problem-based learning, cooperative learning, teacher training, teaching competences 


\section{INTRODUCCIÓN}

En la Carta de Belgrado (1975) se estableció un marco internacional para la Educación Ambiental (EA) y se definió ésta como "el proceso de reconocer valores y clarificar conceptos con el objeto de desarrollar habilidades y actitudes necesarias para comprender y apreciar las interrelaciones entre el ser humano, su cultura y sus entornos biofísicos. La EA incluye también la práctica en la toma de decisiones y la autoformulación de un código de conducta sobre los problemas que se relacionan con la calidad ambiental". Tres décadas después, y ante la gravedad de los problemas a los que todavía se enfrenta el planeta, la ONU proclamó la Década de la Educación para el Desarrollo Sostenible (2005-2014) como "una invitación a repensar nuevas políticas educativas, nuevos programas y nuevas prácticas pedagógicas para que la educación pueda jugar el papel que le concierne en el desarrollo de las capacidades de todos los miembros de la sociedad con el fin de trabajar juntos en la construcción de un futuro durable".

En este sentido, Novo (2006) indica que uno de los retos que debe afrontar la educación del siglo XXI es el reto ambiental, entendido como la capacitación de las personas para reconducir sus ideas y comportamientos hacia una relación armónica con la naturaleza, ya que el incremento de la sensibilidad social hacia la defensa del medio, que se aprecia entre la ciudadanía desde hace unas décadas, no parece haberse traducido en comportamientos sostenibles específicos (Álvarez y Vega, 2009; Gifford, 2014). Las causas de esta situación son complejas, pero es necesario destacar que la EA es un proceso intencional, sostenido en el tiempo, que implica la adquisición de conocimientos conceptuales y valores, así como el desarrollo de actitudes, aptitudes y modos de actuar en interacción social. Tal proceso no puede ser desarrollado en exclusiva, ni de manera aislada, por los sistemas educativos e implica procesos de educación formal y no formal.

Esta problemática ha provocado que se haya señalado la necesidad de orientar la EA hacia el desarrollo de una competencia para la acción, (Ferreira, 2009; Kyburz-Graber, 2013) basada en capacitar al colectivo estudiantil en la adquisición de un pensamiento crítico, que abarque mucho más que conocimientos y actitudes y que contribuya a la formación de una ciudadanía informada y comprometida con el medio y las personas. Este planteamiento apunta hacia un proceso de enseñanza y aprendizaje basado en enfoques democráticos, participativos y orientados hacia la acción que puedan ayudar al alumnado a desarrollar sus habilidades, su motivación y el deseo de jugar un papel activo en la búsqueda conjunta de soluciones a problemas relacionados con el desarrollo sostenible (Mogensen y Schnack, 2010). Nada de ello será posible sin la participación de profesorado comprometido con la educación, que comparta el objetivo de "acción", el uso de métodos pedagógicos poco comunes en clases tradicionales de ciencias, comprenda la naturaleza interdisciplinaria, e incluso globalizada, de las situaciones ambientales y se sitúe en un paradigma crítico.

Este cambio está en consonancia con el nuevo modelo educativo vinculado al desarrollo de competencias, que se está impulsando desde la Unión Europea para responder adecuadamente a las demandas de la sociedad actual. Por su parte, la Agencia Nacional de Evaluación de la Calidad y Acreditación de España (ANECA, 2004) ha establecido las competencias docentes que se deben desarrollar en las distintas titulaciones universitarias. Estas competencias son entendidas como la capacidad de responder a demandas complejas y llevar a cabo tareas diversas de forma adecuada, combinando habilidades prácticas, conocimientos, motivación, valores éticos, actitudes, emociones y otros componentes sociales y de comportamiento que se movilizan conjuntamente para lograr una acción eficaz (OCDE, 2003). Dicha movilización, característica de las competencias, implica la importancia de desarrollar actividades vinculadas a la realidad, es decir, un "aprendizaje situado", la necesidad de interacción social y la puesta en cuestión de modelos exclusivamente basados en la transmisión de conocimientos (Stiefel, 2008).

No es suficiente poseer conocimientos, hay que aprender a movilizarlos, integrarlos y usarlos en diferentes circunstancias de la vida real. Así, las competencias se convierten en logros de aprendizaje, en lugar de una simple adquisición de conocimientos, afectando a los objetivos, al papel del profesorado, a las actividades de enseñanza y a la propia evaluación (Bolívar, 2009). Por ello, su evaluación debe estar centrada en la realización de tareas que estén orientadas hacia la aplicación de saberes adquiridos y hacia la resolución de problemas relacionados con la vida y con los diferentes contextos en los que se desarrolla el alumnado (Vázquez y Ortega, 2012), teniendo en cuenta que adquirir una competencia requiere analizar, reflexionar, emitir juicios, y ajustar su desempeño de acuerdo con las características del marco en que desarrolla su actuación (García et al., 2008).

Este modelo supone un gran reto para el profesorado, sobre todo en el área de Didáctica de las Ciencias experimentales, donde precisa no sólo dominar competencias específicas para llevar a cabo su tarea sino también mantenerse actualizado en los avances científicos y tecnológicos, lo que implica un aprendizaje continuado a lo largo de su carrera profesional. Así, por ejemplo, algunos estudios muestran la inseguridad y el escaso nivel de conocimiento del profesorado de Educación Primaria sobre el medio ambiente o sobre 
estrategias educativas en el campo de la EA, (Forbes y Davis, 2008; Eurydice, 2011). A esto se suma que el profesorado tiende a reproducir los modelos de enseñanza ya experimentados (Garmendia et al., 2014) y que su discurso tiende a ser más innovador que su práctica real (Rodríguez y López, 2006). En este sentido, Arias et al. (2014) apuntan algunas concepciones del futuro profesorado que es urgente cambiar: (a) tiene como marco una metodología tradicional transmisiva, que pone más el acento en enseñar, identificado con explicar, que en aprender, (b) no percibe que el desarrollo de competencias básicas esté relacionado con su función futura ni con la materia estudiada, (c) mantiene para las ciencias una finalidad más centrada en un aspecto cultural, en sentido restrictivo, que en lo relativo a la formación de ciudadanía. Estas ideas contrastan con las prescripciones del Espacio Europeo de Educación Superior, que señalan la necesidad de que la acción docente se centre en el aprendizaje y no en la enseñanza, como ocurría hasta ahora, y pone el acento en el desarrollo de distintas competencias (Bergen, 2005). Reflejan, también, que el alumnado no estima que las ciencias puedan ser una herramienta para la formación de una ciudadanía que participe y actúe para construir un mundo más sostenible y más justo.

Por todo ello, el nuevo modelo educativo basado en competencias implica también un cambio en la formación del profesorado que debe adaptarse a los contextos de aprendizaje actuales y favorecer el desarrollo de las competencias docentes adecuadas (Álvarez-Lires et al., 2013). La formación inicial del profesorado debe promover la reflexión y el desarrollo de profesionales capaces de decidir en contextos y situaciones diferentes de enseñanza y aprendizaje, movilizando conocimientos y habilidades apropiadas, es decir, desarrollar su competencia profesional y el trabajo autónomo (Perrenoud, 2004). Debe, también, favorecer el desarrollo de nuevos puntos de vista entre futuros docentes, diferentes del modelo tradicional, que deben prestar mayor atención al uso de metodologías más activas y participativas. Esto es especialmente relevante en el proceso de enseñanza y aprendizaje de una EA orientada hacia el desarrollo de una competencia para la acción.

De acuerdo con lo expresado anteriormente, el objetivo de este trabajo es explorar la contribución de una intervención didáctica basada en metodologías participativas al desarrollo de algunas de estas competencias específicas en el área de Didáctica de las Ciencias Experimentales, con el fin de contribuir a la formación de profesorado de Educación Primaria reflexivo y comprometido con un futuro sostenible. Concretamente, se estudió la capacidad de alumnado del Grado de Educación Primaria de trabajar en equipo para compartir experiencias y reflexionar sobre la práctica docente y se evaluaron sus conocimientos en un campo temático de interrelación, como es la EA (ANECA, 2004), a través de un planteamiento que también requiere poner en funcionamiento otras competencias de carácter transversal como el tratamiento de la información (transformar dicha información en conocimiento) o la capacidad de análisis y síntesis. La experiencia docente que sustenta esta experiencia se llevó a cabo con alumnado de tercer curso del Grado en Educación Primaria de la Universidad de Vigo, durante el curso académico 201213, en el marco de la asignatura Educación ambiental y su desarrollo. El grupo en cuestión estaba formado por 42 personas, de las cuales el $38 \%$ era de hombres y el $62 \%$ de mujeres.

\section{METODOLOGÍAS PARTICIPATIVAS}

La inclusión en el Espacio Europeo de Educación Superior (Bergen, 2005) implica un profundo cambio en los modelos docentes de las universidades españolas, que deben promover un aprendizaje más autónomo y colaborativo, basados en el desarrollo de competencias como objetivos de aprendizaje. El aprendizaje basado en problemas y el aprendizaje cooperativo responden a estas necesidades; por ello, están siendo impulsados como metodologías que por su carácter participativo y activo favorecen aprendizajes significativos que permiten afrontar situaciones de la vida real.

El aprendizaje basado en problemas, entendido desde el enfoque de una pedagogía de proyectos para el desarrollo de competencias (Zabala, 2014) cuyo antecedente reside en la obra de Kilpatrick (1918), es una estrategia didáctica en la que el alumnado, organizado en grupos, estudia problemas tomados de situaciones reales, con el objetivo de integrar conocimientos y desarrollar habilidades intelectuales de nivel alto. Tuvo su origen en los años 60 para fomentar un aprendizaje multidisciplinar y activo de estudiantes de Medicina, pero actualmente es utilizado en múltiples contextos de aprendizaje, que han constatado que su utilización contribuye a obtener resultados de aprendizaje significativos y promueve el aprendizaje autónomo (Latasa, 2012). Así, el aprendizaje basado en problemas puede ser planteado desde distintas perspectivas en función del proceso de enseñanza y aprendizaje que se desea realizar (Schmidt et al., 2011). Una EA orientada hacia la acción requiere de enfoques globalizadores, orientados a la resolución de problemáticas socioambientales que implique una reflexión y búsqueda de soluciones que consideren a todas las partes implicadas (Álvarez y Vega, 2009). Por ello, en este caso se ha optado por un modelo basado en problemas abiertos (open-ended problems), que se caracterizan porque no es posible resolverlos con absoluta certeza, no pueden caracterizarse completa o unívocamente y generan controversia a la hora de resolverlos (Díaz-Barriga, 2005). Estudios recientes sobre EA muestran la efectividad de planteamientos 
didácticos centrados en la visión comprensiva de un problema y sus posibles soluciones (Darner, 2012; Öhman y Öhman, 2013; Niebert y Gropengiesser, 2013; Varela-Losada et al., 2013), ya que permite al alumnado explicitar sus concepciones, integrar sus conocimientos, tomar decisiones, y, en definitiva, hacer uso de sus competencias.

Esta estrategia puede complementarse a través de organizaciones cooperativas que promueven la construcción de conocimientos a partir de la interacción y la ayuda entre pares, que mejoran procesos cognitivos y preparan a las personas para una vida democrática (Dewey, 1995), ya que este tipo de organización facilita la toma de decisiones en régimen cooperativo, el respeto por la democracia y la comprensión de los procesos de participación (Vega y Álvarez, 2011). Y como señala Novo (2006) el aprendizaje colaborativo y creativo puede servir tanto para construir colectivamente conocimiento como para aplicar esos saberes a la transformación social y a la búsqueda de la equidad. Aunque son muchas las ventajas de utilizar este tipo de metodologías, algunos autores también señalan las dificultades de implementarlas por primera vez. Así, el alumnado puede mostrar estrés o miedo a no alcanzar los objetivos (Garmendia et al., 2014; Vega et al, 2014), no siempre es capaz de desarrollar un aprendizaje autónomo (Prieto et al., 2006) o muestra perspectivas demasiado limitadas a la hora de representar un problema complejo (Öhman y Öhman, 2013).

\section{LA INTERVENCIÓN DIDÁCTICA}

La intervención didáctica realizada está basada en la realización autónoma de tareas a partir de metodologías participativas, principalmente dinámicas cooperativas y aprendizaje basado en problemas, de tal manera que el grupo clase se organizó en todo momento en pequeños grupos de cuatro personas (Schmidt et al., 2011). En todo el proceso tuvo especial protagonismo el trabajo de selección, análisis y organización de la información, con el fin de que el alumnado integrase sus conocimientos iniciales con los nuevos conocimientos necesarios para llevar a cabo la tarea, un pilar fundamental del aprendizaje autónomo.

La secuencia didáctica fue planteada de tal manera que contribuyese a desarrollar de forma socioconstructivista y globalizada las competencias que se han indicado anteriormente. Se establecieron varias fases, en las que se abordaron contenidos ambientales, así como aspectos didácticos y metodológicos relacionados con la Educación Ambiental. Para ello, se realizó una fase de evaluación inicial donde, a través de un formulario Knowledge and Prior Study Inventory (KPSI) (Tamir y Lunetta, 1978) sobre los contenidos de la materia, y de un debate grupal sobre las respuestas a dicho formulario, se dieron a conocer las ideas previas del alumnado, se hicieron explícitas sus necesidades de aprendizaje (Sanmartí, 2007), y se intentó despertar motivación por aprender aquello que se desconoce.

La siguiente fase consistió en la elaboración de un artículo para su publicación en una revista científica sobre Educación Ambiental, a partir de documentación facilitada por el profesorado y de una búsqueda documental guiada. El texto debía incluir una revisión de los objetivos y metodologías recomendadas para esta materia, así como su contraste con el currículo de Educación Primaria. Esta etapa tenía como fin proporcionar al alumnado un marco teórico que le facilitase una reflexión crítica posterior sobre el planteamiento de tareas referentes a temáticas ambientales.

Para ampliar los conocimientos del alumnado sobre contenidos conceptuales medioambientales se realizó una dinámica cooperativa compleja, la técnica del jigsaw o rompecabezas modificada (Slavin, 1990), donde cada estudiante recibe una parte o pieza de una tarea y ha de compartirla con el resto del grupo para poder realizar la actividad con éxito. Para ello, se utilizó un rompecabezas de textos sobre el funcionamiento de un ecosistema, donde cada integrante debía convertirse en un experto de su tópico para luego poder explicárselo correctamente al resto del grupo. La evaluación de esta actividad, diseñada para favorecer la interdependencia positiva del equipo, consistió en un cuestionario final, que sólo debía realizar un componente del grupo elegido al azar, y valoraba el conocimiento global. Tras esta actividad introductoria, se les planteó una tarea de investigación, basada en el estudio de un problema ambiental relacionado con los efectos del cambio de temperatura en un ecosistema. Se optó por un modelo de aprendizaje basado en problemas de carácter abierto (open-ended problems), desarrollado en cuatro fases adaptadas de DíazBarriga (2005) y Prieto et al. (2006), descritas en la Tabla 1, donde el propio alumnado identificó sus necesidades de aprendizaje para llevar a cabo el estudio del problema planteado.

Esta tarea requería que el alumnado aplicase sus conocimientos para analizar la naturaleza multicausal de un problema ambiental, para evaluar los efectos del Cambio Climático en los distintos niveles de organización de un ecosistema y, posteriormente, para relacionar dicho problema con los estilos de vida de nuestra sociedad de consumo. En el desarrollo de esta tarea cada grupo debía responder a una pregunta motriz similar pero situada en distintos contextos (los distintos biomas del planeta), con el fin de que las 
conclusiones fuesen diferentes en cada equipo y que al finalizar la tarea todo el alumnado pudiese reflexionar sobre los efectos del problema en todo el planeta. Este aprendizaje basado en problemas constó de diferentes etapas en las que el alumnado y el profesorado debían cumplir una serie de funciones, que están descritas en la Tabla 1.

Tabla 1: Desarrollo de la experiencia de aprendizaje basado en problemas (open-ended problems), desglosada en cuatro fases. Adaptado de Prieto et al., 2006, y Díaz-Barriga, 2005

\begin{tabular}{|c|c|c|c|}
\hline \multicolumn{2}{|l|}{ Fase } & \multirow{2}{*}{$\begin{array}{l}\text { Rol del Profesorado } \\
\text { - Presenta el problema }\end{array}$} & \multirow{2}{*}{$\begin{array}{l}\text { Rol del Alumnado } \\
\text { - Reparte las funciones } \\
\text { (coordinación, secretaría,...) } \\
\text { - Analiza de forma grupal el } \\
\text { problema }\end{array}$} \\
\hline $\begin{array}{l}\text { 1.Presentación } \\
\text { del problema } \\
\text { motriz }\end{array}$ & $\begin{array}{l}\text { - ¿Qué ocurriría en un ecosistema } \\
\text { situado en un bioma determinado } \\
\text { (desierto, tundra, selva...) si } \\
\text { aumentase la temperatura media } \\
\text { en unos grados? }\end{array}$ & & \\
\hline $\begin{array}{l}\text { 2.Identificación } \\
\text { de las } \\
\text { necesidades } \\
\text { de aprendizaje }\end{array}$ & $\begin{array}{l}\text { - Evolución histórica de la } \\
\text { temperatura y las causas de su } \\
\text { aumento } \\
\text { - Caracterización de cada } \\
\text { ecosistema } \\
\text { - Efecto del aumento de la } \\
\text { temperatura en los diferentes } \\
\text { niveles ecológicos de } \\
\text { organización de la materia y en } \\
\text { las actividades humanas } \\
\text { - Relación con nuestros estilos de } \\
\text { vida }\end{array}$ & $\begin{array}{l}\text { - Supervisa y orienta la } \\
\text { identificación de los } \\
\text { elementos del problema } \\
\text { - Consensua fechas de } \\
\text { entrega }\end{array}$ & $\begin{array}{l}\text { - Participa en una tormenta de } \\
\text { ideas con toda la clase para } \\
\text { identificar elementos del } \\
\text { problema } \\
\text { - Reparte tareas a cada } \\
\text { integrante del equipo y concreta } \\
\text { una planificación con los tiempos } \\
\text { marcados }\end{array}$ \\
\hline $\begin{array}{l}\text { 3. Investigación } \\
\text { y análisis de la } \\
\text { información }\end{array}$ & $\begin{array}{l}\text { - Revisión de la literatura en } \\
\text { diferentes formatos sobre el } \\
\text { cambio climático y su efecto en } \\
\text { los distintos ecosistemas } \\
\text { - Experimentación en laboratorio } \\
\text { sobre el efecto de la temperatura } \\
\text { y la escasez de agua en algunos } \\
\text { seres vivos }\end{array}$ & $\begin{array}{l}\text { - Facilita y orienta la } \\
\text { búsqueda y el análisis } \\
\text { crítico de información } \\
\text { - Proporciona } \\
\text { retroalimentación } \\
\text { - Plantea e inicia } \\
\text { actividades prácticas } \\
\text { relacionadas }\end{array}$ & $\begin{array}{l}\text { - Busca y analiza de forma crítica } \\
\text { la información } \\
\text { - Discute, selecciona y sintetiza la } \\
\text { información de forma grupal } \\
\text { - Organiza la información y la } \\
\text { pone por escrito }\end{array}$ \\
\hline $\begin{array}{l}\text { 4.Conclusiones } \\
\text { y evaluación }\end{array}$ & $\begin{array}{l}\text { - Realización de un informe y } \\
\text { presentación oral de las } \\
\text { conclusiones } \\
\text { - Discusión y reflexión sobre el } \\
\text { problema y sus soluciones } \\
\text { - Evaluación compartida }\end{array}$ & $\begin{array}{l}\text { - Dirige la discusión y } \\
\text { reflexión en gran grupo } \\
\text { - Evalúa el informe y la } \\
\text { presentación }\end{array}$ & $\begin{array}{l}\text { - Presenta a toda la clase sus } \\
\text { conclusiones } \\
\text { - Reflexiona sobre lo que ha } \\
\text { aprendido } \\
\text { - Autoevalúa su trabajo en equipo } \\
\text { - Evalúa las presentaciones de } \\
\text { otros grupos }\end{array}$ \\
\hline
\end{tabular}

La última etapa de la secuencia didáctica consistió en un análisis de la acción realizada, con el fin de que el alumnado efectuase una reflexión fundamentada sobre el proceso. Se les pidió que reflexionasen sobre la posibilidad de emplear un planteamiento similar en aulas de Educación Primaria. Para esta tarea, usaron como criterios de análisis sus propias conclusiones sobre los objetivos y las metodologías más apropiadas para abordar la EA y su relación con los elementos prescriptivos del currículo de Educación Primaria. A lo largo de todo el proceso se utilizó una evaluación formativa y formadora (Sanmartí, 2007), de tal manera que profesorado y alumnado compartieron el proceso evaluativo. Se partió de una evaluación inicial y a lo largo del proceso se utilizaron técnicas de autoevaluación y de evaluación por pares; únicamente el informe y el análisis de la actividad fueron valorados por el profesorado en exclusiva. Con el fin de ayudar al alumnado a conocer e interiorizar los diferentes criterios de evaluación se utilizaron rúbricas específicas (Urbieta et al, 2011), en cuyo diseño y construcción participó el alumnado.

\section{METODOLOGÍA DE LA INVESTIGACIÓN}

Se planificó una intervención didáctica planteada como una investigación-acción. Este tipo de investigaciones, de gran tradición en formación del profesorado (Romera, 2011), tiene como último fin favorecer la mejora continua del proceso de enseñanza y aprendizaje a través de un proceso de investigación del propio grupo y de la interacción durante el propio proceso. La evaluación del proceso se llevó a cabo utilizando una metodología cualitativa a partir de un conjunto de datos de diferente índole (Tabla 2). Los datos se trataron mediante análisis de contenido y codificación de la información. Posteriormente, se realizó un análisis estadístico descriptivo.

Tabla 2: Técnicas de recogida de datos en el proceso 


\begin{tabular}{|c|c|}
\hline Etapas & Técnicas de Recogida de Datos \\
\hline 1․․ Evaluación inicial & $\begin{array}{l}\text { KPSI } \\
\text { Observación directa del debate grupal inicial }\end{array}$ \\
\hline $2^{\circ}$. Realización de un artículo & Producción del alumnado (artículo) \\
\hline $\begin{array}{l}\text { 3․ Rompecabezas sobre contenidos } \\
\text { ecológicos }\end{array}$ & $\begin{array}{l}\text { Observación directa del desarrollo de la dinámica cooperativa } \\
\text { Evaluación grupal (a través de un cuestionario) }\end{array}$ \\
\hline $\begin{array}{l}\text { 4. Intervención didáctica usando } \\
\text { Aprendizaje basado en problemas }\end{array}$ & $\begin{array}{l}\text { Observación directa del desarrollo del proceso de Aprendizaje } \\
\text { basado en problemas } \\
\text { Producción del alumnado (informes + presentaciones orales) } \\
\text { Rúbrica de la presentación (evaluación por pares) }\end{array}$ \\
\hline 5․ Análisis de la propuesta & $\begin{array}{l}\text { Producción del alumnado (informe) } \\
\text { Cuestionario de incidencias críticas }\end{array}$ \\
\hline 6‥ Evaluación del proceso & $\begin{array}{l}\text { Observaciones directas de todo el proceso } \\
\text { Producciones realizadas por el alumnado } \\
\text { Cuestionario de incidencias críticas } \\
\text { Rúbrica de autoevaluación del trabajo cooperativo }\end{array}$ \\
\hline
\end{tabular}

\section{RESULTADOS Y DISCUSIÓN}

La intervención didáctica fue planteada principalmente para proporcionar al alumnado experiencias docentes sobre EA, que utilizasen metodologías participativas diferentes del modelo tradicional sobre las que pudiesen reflexionar y que abordasen contenidos relacionados con el medio y su problemática. Así, para valorar esta intervención se tuvieron en cuenta diversos aspectos del proceso. En relación con el conocimiento del alumnado sobre el medio ambiente, la evaluación de ideas previas reflejó un nivel inicial bajo (fueron incapaces de definir correctamente un ecosistema, no eran conscientes de los flujos de materia y energía o de las interrelaciones entre sus componentes, y no incluían al ser humano como parte del medio). Se ha de señalar que una gran mayoría procedía de un bachillerato (63\%) de humanidades y ciencias sociales.

Como fase previa al estudio de la problemática ambiental, el alumnado realizó una dinámica cooperativa compleja, un rompecabezas de textos sobre el funcionamiento de un ecosistema, con una evaluación de carácter grupal. Los resultados de esta primera evaluación reflejaron lo que parecía un buen resultado, pero el posterior estudio de un problema ambiental real mostró, en su mayoría, un aprendizaje sólo de carácter declarativo. El problema proporcionado requería que el alumnado buscase y seleccionase información sobre el Cambio Climático y sus efectos en un bioma concreto, clasificase y estructurase dichos efectos y reflexionase sobre el efecto global en el mismo, además de estudiar su relación con las actividades y modos de vida del ser humano. Es decir, debía aplicar sus conocimientos al análisis de un problema real y complejo, donde todos los elementos están interrelacionados, con el objetivo último de que reflexionase sobre sus efectos y las posibles soluciones colectivas de las que el alumnado también puede formar parte.

En la realización de la tarea se observaron ciertos problemas, que el alumnado no siempre fue capaz de resolver de forma autónoma, relacionados con:

i) búsqueda y selección de la información: uso de pocas fuentes o fuentes poco apropiadas,

ii) comprensión, análisis y organización de la información: identificación y clasificación errónea de los efectos de la subida de temperatura en los distintos niveles de organización biológica o dificultades en la aplicación de ideas básicas para explicar dichos efectos, así como el uso confuso de términos tales como adaptación, hábitat o nicho ecológico para explicar los efectos de un cambio brusco de temperatura en la distribución de las especies,

iii) representación compleja de un problema ambiental sistémico y global: presentación parcial del problema o no inclusión del ser humano en el ecosistema, obviando que también éste es una parte implicada en los efectos adversos del Cambio Climático.

A pesar de estas dificultades, los resultados del cuestionario de incidencias críticas (Figura 1) mostraron que más de un $80 \%$ del alumnado percibió que había realizado aprendizajes sobre el funcionamiento de un ecosistema y un $54 \%$ sobre los efectos del Cambio Climático. 


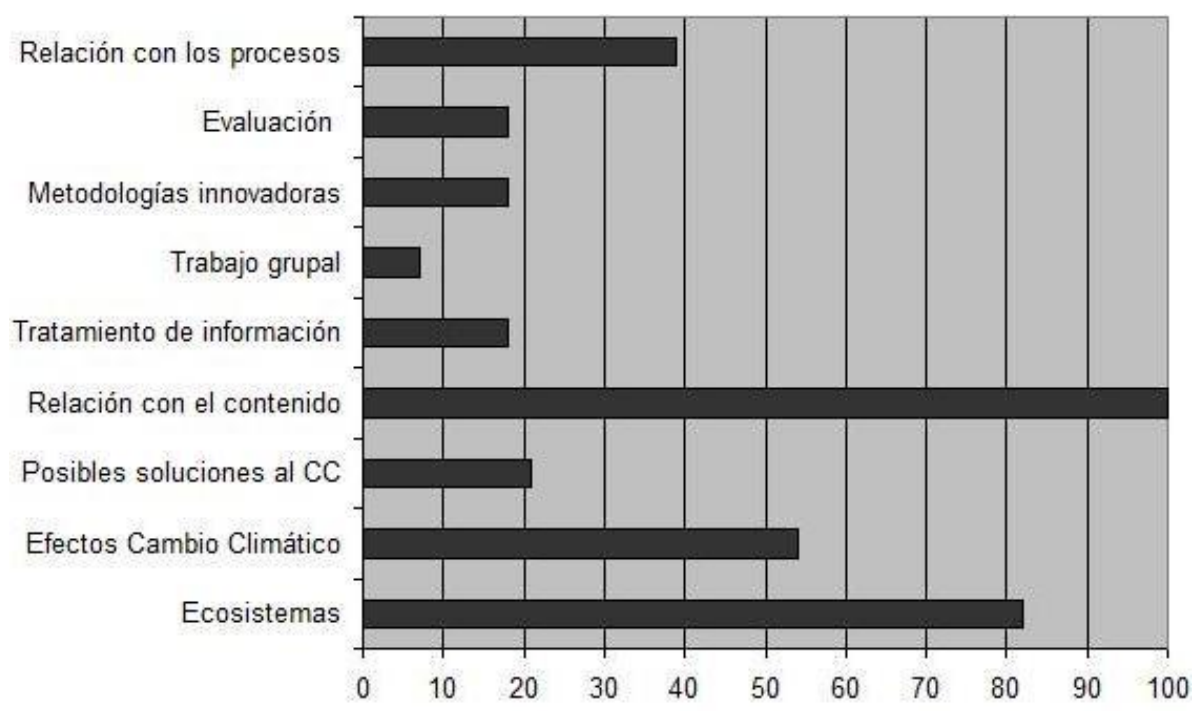

Fig. 1: Respuestas a la pregunta ¿Qué has aprendido? desglosadas en diferentes categorías

La evaluación inicial puso de manifiesto que, aunque durante toda su formación educativa habían trabajado habitualmente de forma grupal, no conocían estrategias para favorecer la colaboración y responsabilidad compartida. Con respecto a los aspectos relacionados con la práctica docente, esta evaluación mostró que el alumnado conocía el currículo de Educación Primaria, aunque mostraba dudas sobre aspectos relacionados con la metodología, la evaluación o las competencias docentes.

La planificación de la secuencia didáctica estaba pensada para que, tras la propia experimentación de una metodología alejada del modelo tradicional transmisivo, el futuro profesorado reflexionase sobre este proceso de enseñanza y aprendizaje. Debían decidir si un planteamiento similar, basado en metodologías participativas, sería adecuado para abordar la EA en la Educación Primaria Con ello, se pretendía que el alumnado aplicase su capacidad de analizar, reflexionar y emitir un juicio razonado sobre el uso de una metodología concreta en un contexto determinado, es decir, aplicar sus conocimientos para analizar un caso real propio de su futuro desempeño profesional. Así, debían realizar una valoración fundamentada teniendo en cuenta aspectos prescriptivos del currículo de Educación Primaria (objetivos, contenidos, competencias básicas) y algunas recomendaciones para la enseñanza de la EA (finalidades, objetivos, metodología) que habían examinado en una fase anterior.

En este análisis, el $100 \%$ de los equipos concluyó que se trataba de un planteamiento didáctico adecuado para llevar a cabo en Educación Primaria, pero la fundamentación de su valoración no se realizó de forma apropiada en su mayoría (Figura 2), ya que el $69 \%$ de los grupos no tuvo en cuenta la totalidad de los ítems propuestos.

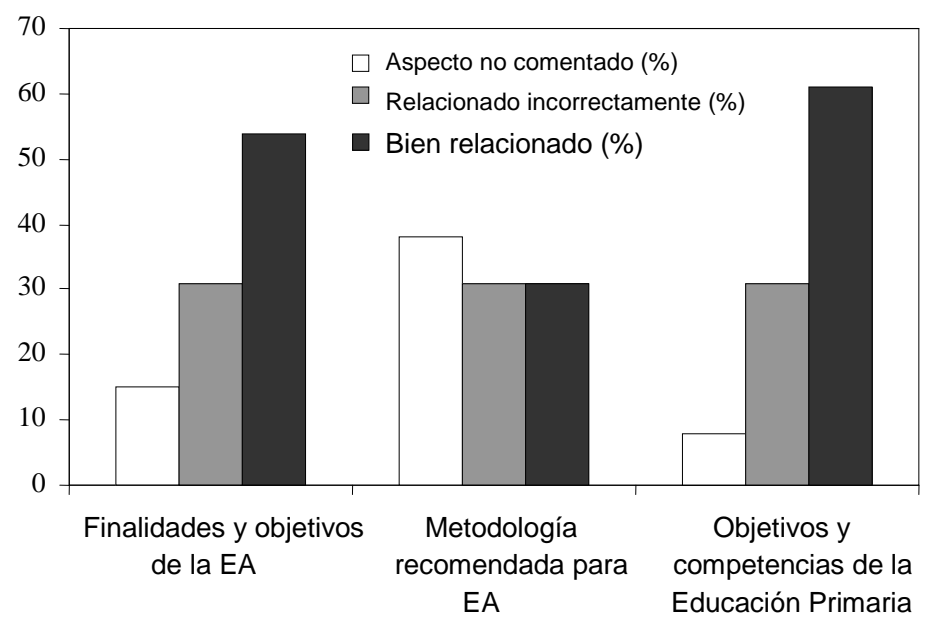

Fig. 2: Aspectos valorados en el análisis de la experiencia 
Los ítems relacionados con la contribución a la consecución de objetivos, contenidos y competencias de la Educación Primaria fueron los que obtuvieron mayor éxito, ya que casi la totalidad de los equipos incluyó estos aspectos, y el $61 \%$ lo hizo correctamente. Con respecto a los ítems relacionados con la Educación Ambiental, hay que señalar que el $85 \%$ utilizó correctamente como criterio de análisis el logro de sus finalidades y objetivos. Sin embargo, se observó una menor tendencia del alumnado a utilizar la metodología como un factor importante de análisis, ya que el 38\% no tuvo en cuenta las recomendaciones sobre metodología de EA o no lo hizo de forma correcta (31\%). Además, en pocos casos se subrayó la pertinencia de utilizar metodologías participativas en esta etapa educativa, que concuerda perfectamente con las recomendaciones del currículo de Primaria en España. Estos resultados sugieren que el alumnado interiorizó parcialmente la importancia del uso una metodología específica en el aprendizaje de la EA y que cuando se le pide que reflexione sobre la práctica educativa no siempre es capaz de analizar y relacionar correctamente los aspectos fundamentales de dicha práctica.

En cuanto a la capacidad de trabajar en grupo, mediante organizaciones cooperativas, la observación directa mostró que el alumnado mantuvo una actitud activa, participativa y comprometida, impresión que también reafirmaron los resultados del cuestionario de incidencias críticas y de la rúbrica de autoevaluación del trabajo cooperativo. No obstante, es necesario señalar que algunos grupos presentaron problemas en el reparto de roles, en la organización de la tarea o en la planificación del tiempo.

La evaluación inicial mostró que el alumnado no conocía ni había experimentado el desarrollo de dinámicas cooperativas ni metodologías de aprendizaje basado en problemas, lo que condicionó algunos resultados de la intervención. En la valoración del proceso se recabó la opinión del alumnado a través de un cuestionario de incidencias críticas, donde se les pedía que valorasen la experiencia, desde la óptica de un futuro docente, a través de cuatro cuestiones semiabiertas, ¿Qué nota de 0 a 10 le pondrían?, ¿Qué habían aprendido? ¿Qué aspectos les habían gustado más del desarrollo de la experiencia de aprendizaje? ¿Qué cambiarían? En su autorreflexión sobre el aprendizaje realizado (Figura 1) se ha de destacar que predominaron los aspectos relacionados con contenidos ambientales conceptuales (el funcionamiento de un ecosistema o el proceso del Cambio Climático) y que sólo un $40 \%$ mencionó algún aspecto relacionado con la metodología u organización de la tarea.

En la valoración general de la experiencia en todos los casos se obtuvo una nota igual o mayor que 7 (media=7,7). Los ítems más destacados como positivos por el alumnado fueron la metodología y organización de la tarea, mencionada, no obstante, solo por un 30\% del alumnado; la distribución en grupos de distintos ecosistemas (30\%), tareas como el rompecabezas (25\%) o la presentación oral (14\%), la búsqueda autónoma de información (18\%), la evaluación por pares (18\%), y en menor medida otros aspectos como su carácter motivador o la mejora del aprendizaje. En este punto debemos destacar que un $70 \%$ del alumnado no mencionó ningún aspecto relacionado con los aspectos metodológicos. En cuanto a los tópicos que el alumnado mencionó como mejorables, cabe destacar que un $77 \%$ apuntó aspectos relacionados con cuestiones técnicas como el aumento en los tiempos de la presentación o la extensión del informe, un $46 \%$ mejoraría temas relacionados con la evaluación (sobre todo mencionaron la necesidad de mejorar el diseño de la rúbrica usada en la evaluación por pares), un $23 \%$ echó en falta una bibliografía inicial o mayor orientación en la búsqueda de la información y un 15\% aconsejó más explicaciones por parte del profesorado. Estos resultados muestran la tendencia de parte del alumnado hacia un modelo tradicional de enseñanza y aprendizaje, que puede estar basado en una concepción de los roles de profesorado y alumnado, y una desconsideración de los aspectos metodológicos, que obstaculizan la posibilidad de aprender a utilizar metodologías innovadoras en enseñanza de las ciencias (Arias et al, 2014)

El desarrollo y del proceso, así como sus resultados, mostraron la necesidad de replantear algunas etapas de la intervención didáctica. La etapa inicial, diseñada como una etapa que debía facilitar la posterior investigación del problema, se mostró claramente insuficiente. El alumnado requería un planteamiento diferente, que le permitiese una reestructuración de sus conocimientos antes de comenzar a estudiar el problema. La puesta en marcha de la etapa del aprendizaje basado en problemas también mostró carencias importantes en la formación en competencias genéricas, tales como la búsqueda y selección información o la capacidad de análisis y síntesis, lo cual indica la necesidad de incluir algunas actividades previas que mejoren su desarrollo. Todo esto, unido a la poca experiencia del alumnado en este tipo de tareas, provocó que en ocasiones el profesorado no sólo proporcionase retroalimentación sino que también interviniese en la resolución de la tarea, generando así un tipo de aprendizaje menos autónomo del que sería deseable. Así, sería preciso evaluar de forma pormenorizada la relación del grupo con el profesorado implicado. Aunque, en este sentido no cabía esperar que en una primera experiencia como la que se llevó a cabo, se pudiese conseguir la autonomía de los aprendizajes, ya que la regulación y autorregulación de dichos procesos es un largo camino. 
También es importante señalar la dificultad que presentó el alumnado para relacionar su aprendizaje con contenidos que no son de carácter conceptual, es decir, con contenidos de carácter procedimental y actitudinal relacionados con el proceso en sí mismo. Y esto es más relevante puesto que se trata de profesorado en formación inicial, que debe prestar especial atención a los procesos de enseñanza y aprendizaje. Este problema, que también se refleja en el desarrollo de su capacidad para reflexionar sobre la práctica docente, apunta la necesidad de explicitar y dedicar mayor atención a estos aspectos en el desarrollo de toda la intervención educativa. La experiencia realizada puede constituir un primer paso para el desarrollo de las competencias indicadas anteriormente.

\section{CONCLUSIONES}

En definitiva, el estudio de esta propuesta educativa muestra que el alumnado fue capaz de trabajar de forma colaborativa y valora positivamente las metodologías innovadoras como el aprendizaje basado en problemas y el aprendizaje cooperativo. Así, con la aplicación de estas metodologías, el alumnado destacó su aprendizaje sobre el funcionamiento de un ecosistema o sobre la comprensión de un problema ambiental complejo.

Pero el proceso también muestra carencias en la formación básica del profesorado, que implica la necesidad de reformular algunas etapas. La realidad detectada es que la mayoría del alumnado posee conocimientos insuficientes sobre el medio ambiente y que estos no siempre están bien estructurados. Esto se traduce en un conocimiento meramente declarativo de las nociones básicas, que unido a su dificultad para utilizar ciertas competencias genéricas relacionadas con la búsqueda, selección y análisis de la información, dificultó el desarrollo de la investigación del problema.

El análisis de la propuesta también parece mostrar la necesidad de mejorar la capacidad de reflexión del alumnado sobre la práctica docente, ya que en algunos casos no logra interiorizar la importancia de utilizar metodologías activas en la enseñanza de la EA o muestra reticencias sobre algunos aspectos de su desarrollo, mostrando aún una tendencia hacia el modelo de enseñanza transmisivo y memorístico.

En definitiva, el análisis inicial de esta propuesta, que forma parte de una investigación todavía en curso, permitió detectar ideas previas y carencias del futuro profesorado, así como su acercamiento a otras metodologías alejadas del modelo tradicional y al desarrollo de competencias profesionales, que tan importantes son para llevar a cabo una EA orientada a la acción.

\section{AGRADECIMIENTOS}

Este trabajo forma parte de un proyecto financiado por el Ministerio de Economía y Competitividad del Gobierno de España (EDU2012-38022-C02-01) sobre el desarrollo de prácticas y competencias.

\section{REFERENCIAS}

Agencia Nacional de Evaluación de la Calidad y Acreditación. Libro blanco del título de grado de Magisterio. Madrid, España (2004).

Álvarez, P. y Vega, P. Actitudes ambientales y conductas sostenibles. Implicaciones para la educación ambiental. Psicodidáctica, 14(2), 245-260 (2009).

Álvarez Lires, M., Arias Correa, A., Pérez Rodríguez, U. y Serrallé Marzoa, J.F. La historia de las ciencias en el desarrollo de competencias científicas. Enseñanza de las Ciencias, 12 (1), 213-233 (2013).

Bolívar, A. Deseñar e avaliar por competencias na universidade. O EEES como reto. Vicerreitoría de Formación e Innovación Educativa, Universidade de Vigo, Vigo, España, (2009).

Carta de Belgrado. Seminario Internacional de Educación Ambiental. Belgrado (1975)

Darner, R. An empirical test of self-determination theory as a guide to fostering environmental motivation, Environmental Education Research 18 (4): 463-472 (2012)

Dewey, J. Democracia y educación, Morata, Madrid, España (1995).

Díaz-Barriga, F. Enseñanza situada: Vínculo entre la escuela y la vida. México: McGraw Hill (2005).

Eurydice. La enseñanza de las Ciencias en Europa: políticas nacionales, prácticas e investigación, Agencia Ejecutiva en el ámbito educativo, audiovisual y cultural, Bruselas (2011)

European Higher Education Area. Comunicado de Bergen. Achieving the goals. Bergen. 19 - 20 Mayo (2005). 
Ferreira, J. Unsettling orthodoxies: Education for the environment for sustainability. Environmental Education Research 5(5):607-620 (2009).

Forbes, C. T. y Davis, E. A. Exploring preservice elementary teachers' critique and adaptation of science curriculum materials in respect to socioscientific issues. Science \& Education, 17, 829-854 (2008).

García, B., Loredo, J., Luna, E., y Rueda, M. Modelo de evaluación de competencias docentes para la educación media y superior. Revista Iberoamericana de Evaluación Educativa, 1(3), 97-108 (2008).

Garmendia, M., Fuentes, J. I. B., Elosegi, K. Z., y Aranzábal, J. G. Proyecto de formación del profesorado universitario de Ciencias, Matemáticas y Tecnología, en las metodologías de Aprendizaje Basado en Problemas y Proyectos. Enseñanza de las ciencias, 32(2), 113-129 (2014)

Gifford, R. Environmental psychology matters. Psychology, 65(1): 541-579 (2014)

Kilpatrick, W. The project method. The Teachers College Record, 19(4), 319-335 (1918)

Kyburz-Graber, R. Socioecological Approaches to Environmental Education and Research. In International Handbook of Research on Environmental Education, editado por Stevenson R.B., Brody, M., Dillon, J. y Wals, A.E.J. New York : Routledge (2013)

Latasa, I., Lozano, P., y Ocerinjauregi, N. Aprendizaje Basado en Problemas en Currículos Tradicionales: Beneficios e Inconvenientes. Formación universitaria, 5(5), 15-26 (2012).

Mogensen F. y K. Schnack. The action competence approach and the 'new' discourses of education for sustainable development, competence and quality criteria. Environ. Educ. Research 16 (1):59-74 (2010)

Niebert, K. y H. Gropengiesser. Understanding and communicating climate change in metaphors, Environmental Education Research 19 (3): 282-302 (2013)

Novo, M. El desarrollo sostenible, Pearson educación, Madrid, España (2006).

OCDE. Definición y selección de competencias clave. Resumen ejecutivo. Organización para la Cooperación y el Desarrollo Económico, (2003).

Öhman, J. y Öhman, M. Participatory approach in practice: an analysis of student discussions about climate change, Environmental Education Research 19(3): 324-341 (2013)

Perrenoud, P. Cuando la escuela pretende preparar para la vida: ¿ Desarrollar competencias o ensenar otros saberes? Barcelona. Grao.(2012)

Prieto Martín, A. y otros seis autores, Un nuevo modelo de aprendizaje basado en problemas, el ABP $4 \times 4$, es eficaz para desarrollar competencias profesionales valiosas en asignaturas con más de 100 alumnos. Aula abierta, 87, 171-194 (2006)

Romera-Iruela, M. J. La investigación-acción en la formación del profesorado. Revista Española de Documentación Científica, 34(4), 597-614 (2011)

Sanmartí, N. 10 ideas clave: evaluar para aprender. Graó, Barcelona, España, (2007).

Schmidt, H. G., Rotgans, J. I. y Yew, E. H. The process of problem-based learning: what works and why. Medical education, 45(8), 792-806 (2011)

Slavin, R. E. Cooperative learning. Theory, research and practice, Allyn and Bacon, (1990).

Stiefel, B. M. Competencias básicas: hacia un nuevo paradigma educativo. Narcea, Madrid, España (2008)

Tamir, P., y Lunetta, V. N. An Analysis of Laboratory Inquiries in the BSCS Yellow Version. American Biology Teacher, 40(6), 353-7 (1978)

Vega Marcote, P. y P. Álvarez Suárez. La Agenda 21 y la huella ecológica como instrumentos para lograr una universidad sostenible. Enseñanza de las ciencias, 29(2), 207-217 (2011).

Urbieta, J. M. E., Garayalde, K. A., y Losada, D. Diseño de rúbricas en la formación inicial de maestros/as. Revista de Formación e Innovación Educativa Universitaria, 4(3), 156-169 (2011).

Varela-Losada, M; Álvarez-Lires, M., Pérez-Rodríguez, U. y Serrallé, J.F. El aprendizaje basado en problemas como propuesta didáctica de EA para la sostenibilidad en formación inicial del profesorado. Enseñanza de las Ciencias, Num.extra, 3618-3623 (2013).

Vázquez, P. y Ortega, J. Competencias básicas, Wolters Kluwer, Madrid, España (2012).

Vega, F.; Portillo, E.; Cano, M. y Navarrete, B. Experiencias de aprendizaje en ingeniería química: diseño, montaje y puesta en marcha de una unidad de destilación a escala laboratorio mediante aprendizaje basado en problemas. Form. Univ., 7(1), 13-22 (2014)

Zabala, A. Métodos para la enseñanza de las competencias. Graò, Barcelona, España (2014) 